\title{
ANÁLISE DOS CICLOS DE TENSÕES EM COLHEDORAS DE FORRAGENS
}

\section{PEDRO H. M. BORGES ${ }^{1}$, ORLANDO K. NIIYAMA ${ }^{2}$, ROBERTO A. BRAGA JÚNIOR ${ }^{3}$, CARLOS E. S. VOLPATO ${ }^{3}$, GIOVANNI F. RAVELO ${ }^{3}$, LUCIANO M. DOS SANTOS ${ }^{4}$}

\begin{abstract}
RESUMO: O estudo das tensões em colhedoras de forragens é imprescindível, uma vez que as condições de operação são muito variáveis e severas. Utilizou-se um sistema "datalogger" devidamente calibrado para medir as tensões, em função do tempo, numa colhedora de forragem. Foram desenvolvidos modelos matemáticos para obter a variação periódica dessas tensões, considerando-se a influência da roda de apoio e da velocidade angular do rotor de corte. O ajuste dos modelos foi verificado pelo coeficiente de determinação $\left(\mathrm{R}^{2}\right)$. A variação periódica das tensões, em função do tempo, para os valores experimentais e teóricos, foi representada graficamente, utilizando-se da planilha eletrônica EXCEL. Observou-se que as menores amplitudes de tensões e os maiores períodos do ciclo corresponderam aos tratamentos que utilizaram a roda de apoio. A análise estatística mostrou que a velocidade angular do rotor de corte apresentou diferença significativa, conforme o Teste de Tukey, para 5\%. Com base nos resultados obtidos, concluiu-se que os ciclos de tensões em colhedoras de forragens podem ser representados com bom ajuste por funções senoidais e que o uso da roda de apoio pode aumentar o tempo de serviço da colhedora.
\end{abstract}

PALAVRAS-CHAVE: colhedora de forragem, tensões, modelos matemáticos.

\section{ANALYSIS OF STRESS CYCLE IN FORAGE HARVESTERS}

SUMMARY: The study of stress in forage harvester is necessary, because the operation conditions are very different and rigorous. A calibrated datalogger system was used to measure the stress as function of time for a forage harvester. Mathematical models were developed to obtain the periodical variation of this stress by considering the influence of support wheel and the angular velocity of cutterheads. The agreement of these models was verified by the determination coefficient $\left(\mathrm{R}^{2}\right)$. The periodical variation of stress as function of time for both experimental and theoretical values was graphically represented by using of the software EXCEL. It was observed that the minimal amplitude stress and the maximal periods of cycle corresponded to the treatments employed, which utilized the support wheel. The statistical analysis showed that the angular velocity of cutterheads presented significant difference, according to the Tukey Test for 5\%. Based on the obtained results was concluded that, the stress cycle can be represented by senoidal functions with an adequate agreement, and that the operation time of harvesters can be increased by using a support wheel.

KEYWORDS: forage harvester, stress, mathematical models.

\footnotetext{
${ }^{1}$ Prof. Doutor, Departamento de Engenharia, Universidade Federal de Lavras, Lavras - MG, Fone: (0XX35) 3829.1668, e-mail: hurtado@ufla.br

${ }^{2}$ Bolsista de Iniciação Científica do CNPq-PIBIC, Universidade Federal de Lavras, Lavras - MG.

${ }^{3}$ Prof. Doutor, Departamento de Engenharia, Universidade Federal de Lavras, Lavras - MG.

${ }^{4}$ Eng ${ }^{\mathrm{o}}$ Agrícola, Departamento de Engenharia, Universidade Federal de Lavras, Lavras - MG.

Recebido pelo Conselho Editorial em: 28-8-2002

Aprovado pelo Conselho Editorial em: 26-7-2004
} 


\section{INTRODUÇÃO}

No processo da colheita de forragem, há necessidade de corte, carregamento e envio do material cortado e picotado para o meio de transporte, daí a importância das colhedoras, que são máquinas destinadas a esse fim. Segundo GARCIA et al. (1998), grande parte das colhedoras de forragens em uso no Brasil foram desenvolvidas por tentativas e erros, sem passarem por um processo de otimização de seu projeto. Por essa razão, são vários os pontos que merecem atenção por parte dos engenheiros projetistas, entre os quais a influência das vibrações na vida útil destas máquinas. Diversos trabalhos, tais como: OLIVEIRA (1991), OLIVEIRA (1997), SANTOS (1998) e SILVA \& OLIVEIRA (1998) tiveram como finalidade principal estudar a influência das vibrações de máquinas agrícolas sobre os operadores, em função das condições de trabalho. Por outro lado, BORGES (1991 e 1994) desenvolveu equações para estimar as demandas de força, energia e potência nas colhedoras com tambor picador de facas radiais fixas. No entanto, nessas pesquisas, não foram relatadas informações referentes a conseqüências negativas que poderiam ser ocasionadas pelas vibrações presentes nos mecanismos e/ou sistemas que compõem as máquinas agrícolas.

O movimento dos mecanismos e peças que compõem as colhedoras de forragens origina tensões nos seus elementos mecânicos, que variam periodicamente. Isso significa uma provável falha por fadiga, resultado da aplicação contínua de cargas alternadas, pulsativas e randômicas. Assim, ocorre diminuição do tempo de serviço da máquina. Grande número de experiências mostra tempos de serviço para máquinas praticamente infinitos, independentemente do número de vezes que a carga seja "pulsada", quando as tensões desenvolvidas no elemento se encontram abaixo de determinado valor (ALBURQUERQUE, 1980). Em geral, de acordo com DOBROVOLSKI et al. (1976), FRONIUS (1979), HARTOG (1972), SCHLOTTMANN (1979), TAVARES (1999) e WÄCHTER (1987), o projeto de um elemento mecânico é baseado nos parâmetros de resistência mecânica obtidos no diagrama tensão-deformação. Dependendo do coeficiente de segurança empregado, os componentes mecânicos podem ter rigidez necessária para resistir aos carregamentos e/ou solicitações estáticas sem fraturar. Contudo, esses componentes podem sofrer fraturas, mesmo com carregamentos inferiores aos especificados no projeto estático. Essa situação é decorrente da não-consideração da variação periódica de tensões que surgem durante o serviço da máquina.

Este trabalho tem como objetivo fazer uma análise das tensões oscilatórias em colhedoras de forragens, máquinas essas que operam em condições de carregamento variáveis e severas, e são amplamente utilizadas na agricultura. Nesta análise, pretende-se determinar a influência da variação periódica das tensões no tempo de serviço das referidas máquinas.

\section{MATERIAL E MÉTODOS}

O trabalho foi desenvolvido nos Laboratórios de Máquinas e Implementos Agrícolas e de Automação e Controle do Departamento de Engenharia da UFLA. Os equipamentos necessários para realizar os testes foram:

1. Trator agrícola de rodas modelo VALMET 68, cujas características técnicas, conforme o fabricante são:

- Potência no motor a 2.200 rpm: 53,7 kW (73 cv);

- Potência máxima na tomada de potência (TDP): 48,3 kW (65,7 cv);

- Potência na tomada de potência (TDP) a $540 \mathrm{rpm}: 42,63 \mathrm{~kW}(58 \mathrm{cv})$.

2. Colhedora de forragem modelo JF $90 \mathrm{Z} 10$ (Figura 1), cujas características técnicas, conforme o fabricante, são:

- Potência mínima na tomada de potência (TDP) do trator: 40,43 kW (55 cv); 
- Número de rolos: 4;

- Produção: 20 a $30 \mathrm{t} \mathrm{h}^{-1}$;

- Número de facas do rotor: 10;

- Rotação na tomada de potência (TDP): 540 rpm;

- Rotação no rotor de corte: $1.450 \mathrm{rpm}$;

- Opções de corte: $2 ; 3 ; 5 ; 6,5 ; 8,5$ e $11 \mathrm{~mm}$;

- Massa (com roda de apoio e dispositivo de giro): $579 \mathrm{~kg}$;

- Massa da roda de apoio: $13 \mathrm{~kg}$.

3. Sistema de aquisição automática de dados (“datalogger”), DC-100;

4. Extensômetro elétrico de resistência ("strain gages"), e

5. Cronômetro.

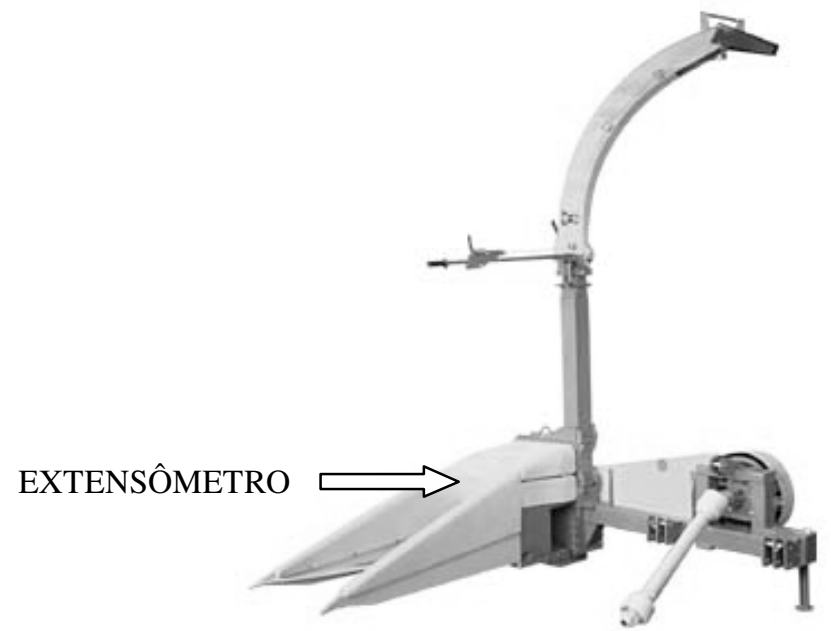

FIGURA 1. Colhedora de forragem JF 90 Z 10.

As engrenagens do sistema de transmissão da colhedora foram selecionadas para obter a mínima e a máxima velocidade angular do rotor de corte. Durante o ensaio, foi considerada, ainda, a colocação da roda de apoio. Assim, foram avaliados os quatro tratamentos $\left(\mathrm{T}_{\mathrm{i}}\right)$ seguintes:

1. Mínimas rotações sem a roda de apoio $\left(\mathrm{T}_{1}\right)$;

2. Mínimas rotações com a roda de apoio $\left(\mathrm{T}_{2}\right)$;

3. Máximas rotações sem a roda de apoio $\left(\mathrm{T}_{3}\right)$, e

4. Máximas rotações com a roda de apoio $\left(\mathrm{T}_{4}\right)$.

O extensômetro foi instalado na parte interna da plataforma de corte da colhedora, bem próximo à contrafaca (Figura 1). Com o auxílio do sistema de aquisição de dados e o cronômetro, obteve-se experimentalmente a variação periódica das tensões para cada tratamento $\left(\mathrm{T}_{\mathrm{i}}\right)$, durante 100 segundos. Considerando-se que os valores experimentais das tensões, em função do tempo, não seguem uma determinada função trigonométrica, optou-se por selecionar um ciclo crítico ou representativo para cada tratamento. Esse ciclo corresponde ao intervalo de tempo em que foi registrada a maior diferença entre as tensões mínima e máxima. Com base nos trabalhos de BEER \& JOHNSTON JR. (1996), BRANSON (1974) e HARTOG (1972), os dados experimentais obtidos foram modelados matematicamente, conforme a seguinte função periódica senoidal: 


$$
\sigma(\mathrm{t})=\sigma_{\mathrm{a}} \operatorname{sen}(\omega \mathrm{t}+\phi)-\sigma_{\mathrm{m}}
$$

em que,

$\sigma_{\mathrm{a}}$ - amplitude de tensão do ciclo, $\mathrm{MPa}$;

$\sigma_{\mathrm{m}}$ - tensão média do ciclo, $\mathrm{MPa}$;

$\mathrm{t}$ - tempo, $\mathrm{s}$;

$\omega$ - frequiência angular, $\mathrm{c} \mathrm{s}^{-1}$, e

$\phi$ - ângulo de fase, rad.

Os parâmetros da eq.(1) são calculados como:

$$
\begin{aligned}
& \sigma_{\mathrm{a}}=\left(\sigma_{\max }-\sigma_{\min }\right) / 2 \\
& \sigma_{\mathrm{m}}=\left(\sigma_{\max }+\sigma_{\min }\right) / 2 \\
& \phi=\operatorname{arcsen}\left[\left(\sigma_{\mathrm{o}}-\sigma_{\mathrm{m}}\right) / \sigma_{\mathrm{a}}\right] \\
& \omega=2 \pi / \mathrm{T}
\end{aligned}
$$

em que,

$\sigma_{\min }$ - tensão mínima do ciclo, $\mathrm{MPa}$;

$\sigma_{\max }$ - tensão máxima do ciclo, $\mathrm{MPa}$;

$\sigma_{\mathrm{o}}$ - tensão inicial do ciclo, $\mathrm{MPa}, \mathrm{e}$

$\mathrm{T}$ - período do ciclo, $\mathrm{s}$.

Segundo DRAPER \& SMITH (1981) e VEIGA \& SÁFADI (1999), o ajuste dos modelos desenvolvidos foi verificado usando-se o coeficiente de determinação $\left(R^{2}\right)$, definido pela relação entre os desvios dos valores teóricos e experimentais. Esse coeficiente indica ajuste adequado se o seu valor absoluto for maior que 0,3 e menor que $1\left(0,3<\mathrm{R}^{2}<1\right)$. Para representar graficamente os valores experimentais e teóricos das tensões, em função do tempo, foram utilizados os recursos computacionais da planilha eletrônica EXCEL. Os resultados foram submetidos à análise estatística, com o auxílio do sistema SISVAR, desenvolvido por FERREIRA (2000). As médias dos tratamentos foram comparadas a 5\% de probabilidade, pelo teste de Tukey, de acordo com PIMENTEL GOMES (1990).

\section{RESULTADOS E DISCUSSÃO}

Nas Figuras 2 e 3, apresenta-se a variação periódica dos valores experimentais e teóricos da tensão em função do tempo, para cada tratamento $\left(\mathrm{T}_{\mathrm{i}}\right)$. Dessas figuras, deduz-se que os tratamentos $\mathrm{T}_{2}$ e $\mathrm{T}_{4}$ apresentaram maior intervalo de tempo entre as tensões extremas (mínimas e máximas) do ciclo. Isso significa que a colocação da roda de apoio ocasiona um efeito de amortecimento na colhedora, o que, sem dúvida, influencia positivamente no tempo de serviço dessas máquinas. Nas referidas figuras, verifica-se que os modelos desenvolvidos apresentaram bom ajuste, dado que o coeficiente de determinação obtido para cada tratamento foi superior a 0,7 .

Na Tabela 1, são apresentados os valores das tensões usadas na modelagem matemática para cada tratamento. Nessa tabela, nota-se que os maiores valores das tensões mínima e máxima e, conseqüentemente, os maiores valores da tensão média corresponderam aos tratamentos $\mathrm{T}_{1}$ e $\mathrm{T}_{2}$. Isso concorda com o esperado, uma vez que o aumento da velocidade angular do rotor de corte ocasiona aumento do seu momento de inércia, o que favorece superar as resistências opostas ao movimento desse sistema com menores flutuações das tensões na colhedora. 




- valores experimentais —valores teóricos

(a)

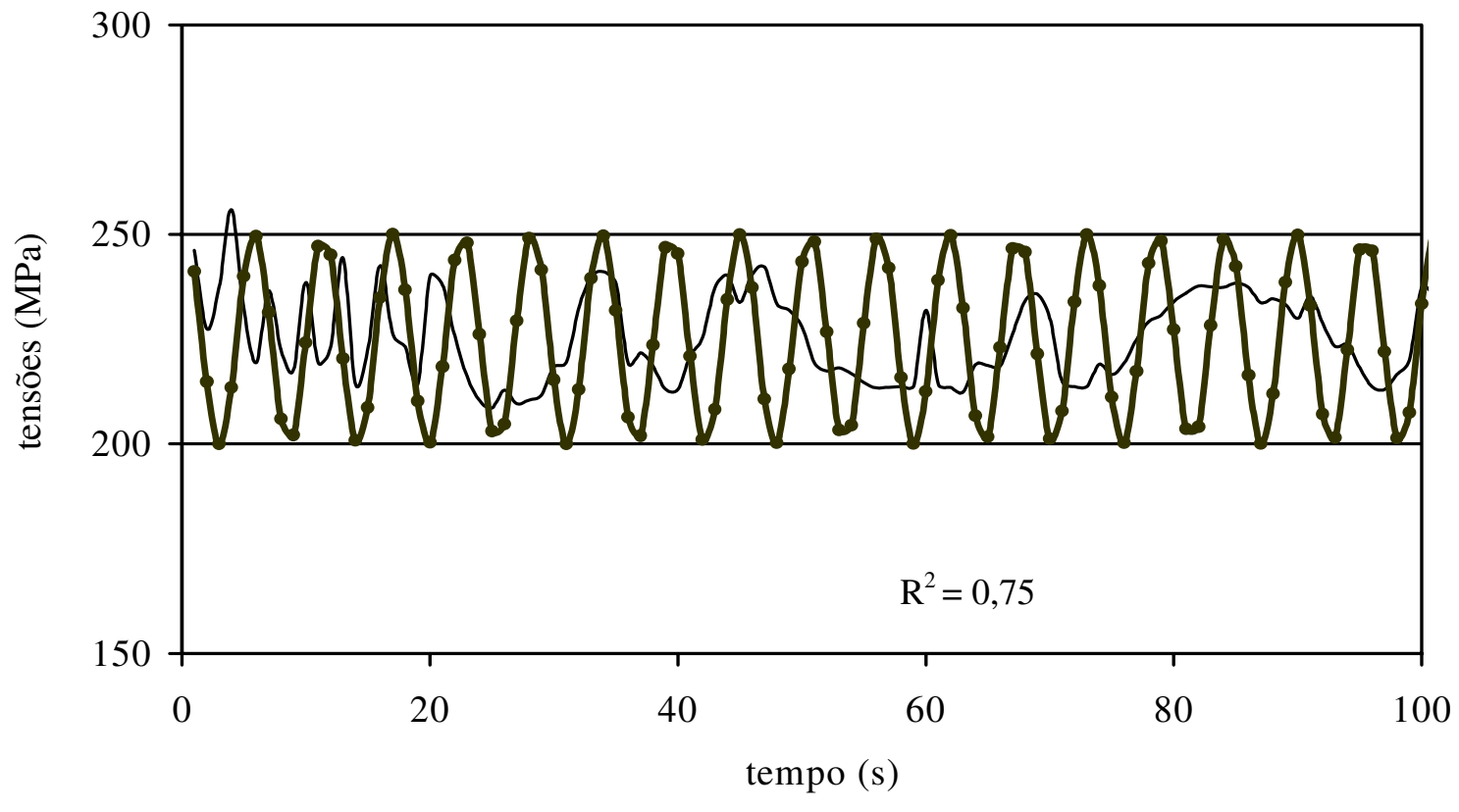

$\longrightarrow$ valores experimentais $\longrightarrow$ valores teóricos

(b)

FIGURA 2. Valores experimentais e teóricos da tensão, em função do tempo, para as mínimas rotações do rotor de corte: (a) sem a roda de apoio, e (b) com a roda de apoio. 


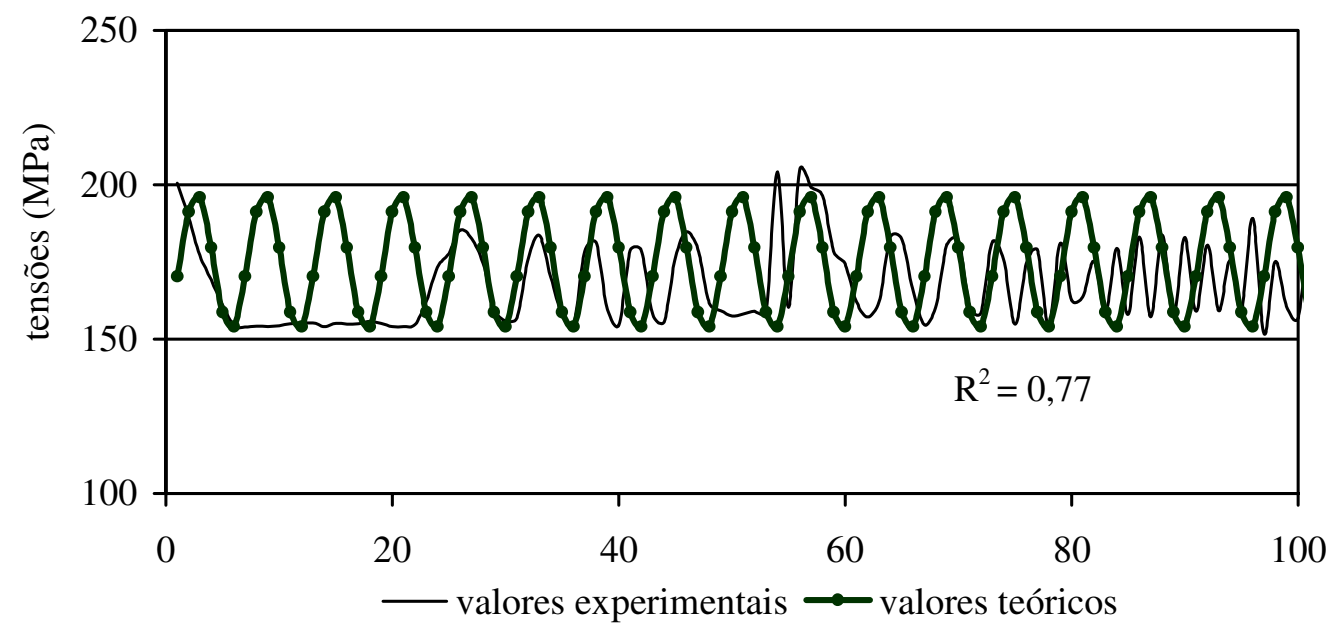

(a)

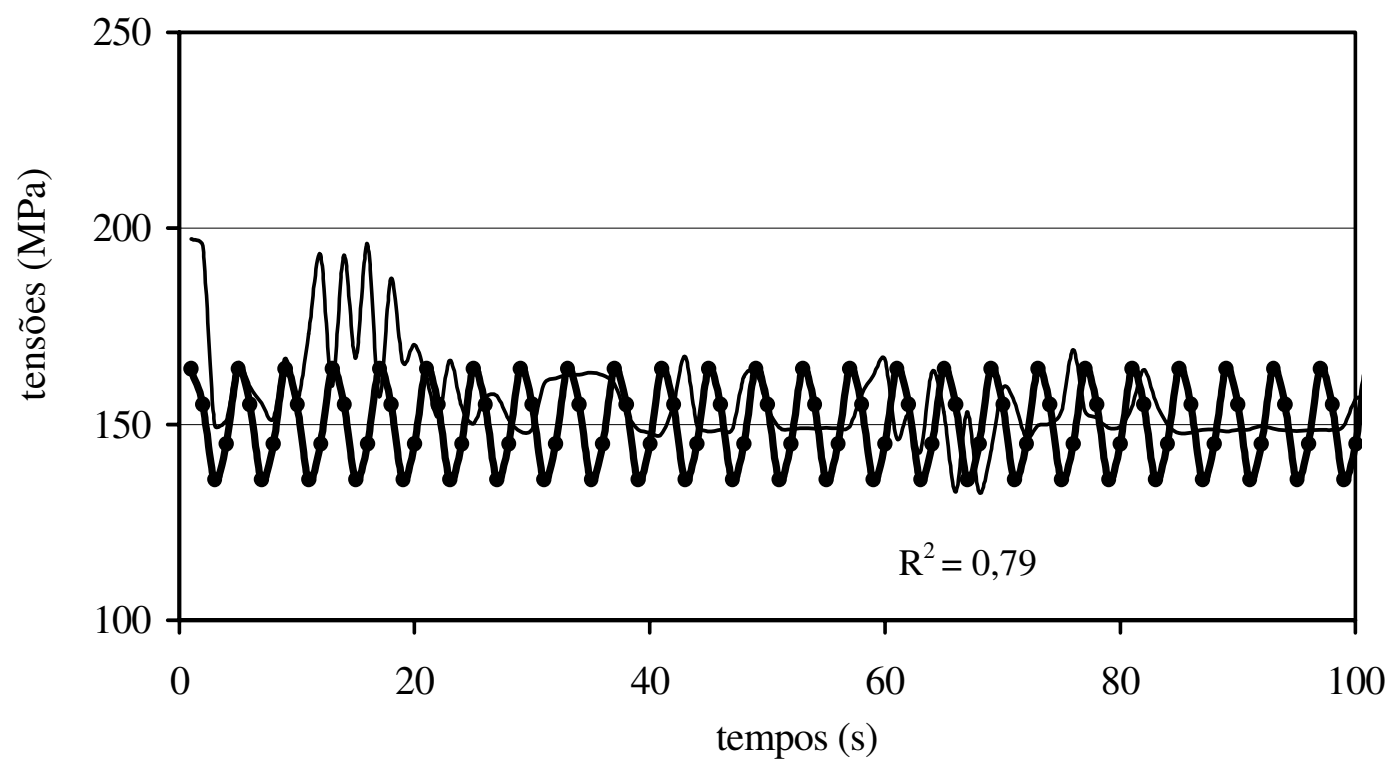

- Valores práticos $\rightarrow$ Valores teóricos

(b)

FIGURA 3. Valores experimentais e teóricos da tensão, em função do tempo, para as máximas rotações do rotor de corte: (a) sem a roda de apoio, e (b) com a roda de apoio. 
TABELA 1. Tensões usadas na modelagem matemática para cada tratamento.

\begin{tabular}{cccccc}
\hline \multirow{2}{*}{ Tratamentos } & \multicolumn{5}{c}{ Tensões (MPa) } \\
\cline { 2 - 6 } & $\sigma_{\mathrm{o}}$ & $\sigma_{\min }$ & $\sigma_{\max }$ & $\sigma_{\mathrm{m}}$ & $\sigma_{\mathrm{a}}$ \\
\hline $\mathrm{T}_{1}$ & 218 & 206 & 240 & 223 & 17 \\
$\mathrm{~T}_{2}$ & 226 & 209 & 235 & 222 & 13 \\
$\mathrm{~T}_{3}$ & 175 & 155 & 185 & 170 & 15 \\
$\mathrm{~T}_{4}$ & 152 & 148 & 166 & 157 & 9 \\
\hline
\end{tabular}

Ainda pode-se constatar que o emprego da roda de apoio reduz a amplitude de tensão em 23,53 e $40 \%$, respectivamente, para as mínimas e as máximas velocidades angulares do rotor de corte (Tabela 1). Pela própria tabela, observa-se que o tratamento $T_{4}$ apresentou a menor amplitude de tensão. Isso significa que o efeito positivo de usar a roda de apoio pode ser aumentado consideravelmente, quando as rotações do rotor de corte são elevadas.

Conforme a Tabela 2, os maiores valores do período do ciclo e, portanto, os menores valores da freqüência angular foram obtidos para os tratamentos $\mathrm{T}_{2}$ e $\mathrm{T}_{4}$. Isso concorda com o esperado, devido ao efeito de amortecimento causado pela roda de apoio. Com base na análise estatística realizada, pode-se verificar que a velocidade angular do rotor de corte evidenciou diferença significativa, de acordo com o teste de Tukey, para 5\%, independentemente do uso da roda de apoio (Tabela 3).

TABELA 2. Período do ciclo e parâmetros do argumento da função senoidal para cada tratamento.

\begin{tabular}{cccc}
\hline Tratamentos & $\begin{array}{c}\text { Período do Ciclo }(\mathrm{T}) \\
(\mathrm{s})\end{array}$ & $\begin{array}{c}\text { Freqüência Angular }(\omega) \\
\left(\mathrm{c} \mathrm{s}^{-1}\right)\end{array}$ & $\begin{array}{c}\text { Ângulo de Fase }(\phi) \\
(\mathrm{rad})\end{array}$ \\
\hline $\mathrm{T}_{1}$ & 5 & 1,2566 & $-0,2985$ \\
$\mathrm{~T}_{2}$ & 10 & 0,6283 & 0,3128 \\
$\mathrm{~T}_{3}$ & 6 & 1,0472 & 0,3398 \\
$\mathrm{~T}_{4}$ & 12 & 0,5236 & $-0,5890$ \\
\hline
\end{tabular}

TABELA 3. Análise estatística das tensões em função do tempo.

\begin{tabular}{ccc}
\hline \multirow{2}{*}{ Velocidade Angular do Rotor de Corte } & \multicolumn{2}{c}{ Tensões (MPa) } \\
\cline { 2 - 3 } & Sem Apoio & Com Apoio \\
\hline Mínima & $224,64 \mathrm{a}$ & $220,22 \mathrm{a}$ \\
Máxima & $159,50 \mathrm{~b}$ & $158,64 \mathrm{~b}$ \\
Média & 192,07 & 189,43 \\
\hline
\end{tabular}

Médias seguidas pela mesma letra nas colunas não diferem estatisticamente (Tukey 5\%).

\section{CONCLUSÕES}

Os ciclos de tensões em colhedoras de forragens podem ser modelados matematicamente com bom ajuste, conforme funções senoidais.

A variação periódica das tensões em colhedoras de forragens foi influenciada pela velocidade angular do rotor de corte.

O uso da roda de apoio reduz a amplitude das tensões, o que, sem dúvida, aumenta o tempo de serviço da colhedora de forragens. 


\section{REFERÊNCIAS}

ALBURQUERQUE, O.A.L.P. Elementos de máquinas. Rio de Janeiro: Guanabara Dois, 1980. 445 p. BEER, F.P.; JOHNSTON JR., E.R. Mecânica vetorial para engenheiros: estática e dinâmica. São Paulo: Makron Books do Brasil, 1996. 1.234 p.

BORGES, P.H.M. Rechnergestützte Berechnungsvorschriften ausgewählter Maschinen-elemente von Trommelhäcksleraggregaten. 1991. 132 f. Tese (Doutorado em Máquinas Agrícolas) - Universidade de Rostock, Rostock, 1991.

BORGES, P.H.M. Determinación de las fuerzas en las cuchillas de los picadores con tambor rotatorio. Construcción de Maquinária, Santa Clara, v.19, n.2, p.3-9, 1994.

BRANSON, L.K. Mecânica: estática e dinâmica. Rio de Janeiro: Livros Técnicos e Científicos, 1974. $430 \mathrm{p}$.

DOBROVOLSKI, V.; ZABLONSKI, K.; MAK, S.; RADCHIK, A. Elementos de máquinas. Moscú: MIR, 1976. $541 \mathrm{p}$.

DRAPER, N.R.; SMITH, H. Applied regression analysis. New York: John Wiley \& Sons, 1981. $709 \mathrm{p}$.

FERREIRA, D.F. Análises estatísticas por meio do Sisvar para Windows versão 4.0. In: REUNIÃO ANUAL DA REGIÃO BRASILEIRA DA SOCIEDADE INTERNACIONAL DE BIOMETRIA, 45., 2000, São Carlos. Anais ... São Carlos: SIB, 2000. p.255-8.

FRONIUS, S. Konstruktionslehre. Antriebselemente. Berlin: Verlag Technik, 1979. 432 p.

GARCIA, R.F.; QUEIROZ, D.M.; DIAS, G.P. Análise de tensões na faca de corte de uma colhedora de forragens. Revista Brasileira de Engenharia Agrícola e Ambiental, Campina Grande, v.2, n.2, p.219-23, 1998.

HARTOG, J.P. Vibrações nos sistemas mecânicos. São Paulo: Edgard Blücher, 1972. 366 p.

OLIVEIRA, N.V. Identificación experimental de fuerzas bajo condiciones controladas en el dominio de la frecuencia. In: PAN AMERICAN CONGRESS FOR APPLIED MECHANICS, 2., 1991, Val Parayso - Chile. Proceedings... Val Parayso: PACAM, 1991. v.1, p.183-8.

OLIVEIRA, N.V. The criterion conjunction utilizing the Nyquist Theorem for the dynamics simulation of the mechanical system. In: BRAZILIAN CONGRESS OF MECHANICAL ENGINEERING, 14., 1997, Bauru. Proceedings... Bauru: ABCM COBEM97, 1997. v.1, p.171. PIMENTEL GOMES, F. Curso de estatística experimental. Piracicaba: Novel, 1990. 468 p.

SANTOS, I.F. Cinemática e dinâmica de sistemas mecânicos: modelagem, simulação, visualização e verificação. Campinas: DPM-FEM-UNICAMP, 1998. 283 p.

SCHLOTTMANN, D. Konstruktionslehre. Grundlagen. Berlin: Verlag Technik, 1979. 428 p.

SILVA, J.C.B.; OLIVEIRA, N.V. Análise das vibrações e identificação de parâmetros em trator de pneu. In: CONGRESSO BRASILEIRO DE ENGENHARIA AGRÍCOLA, 27., 1998, Poços de Caldas. Anais... Lavras: Sociedade Brasileira de Engenharia Agrícola, 1998. v.3, p.259-61.

TAVARES, G. Elementos orgânicos fundamentais de máquinas e implementos agrícolas. Londrina: UEL, 1999. 247 p.

VEIGA, R.D.; SÁFADI, T. Análise de regressão e séries temporais. Lavras: UFLA, 1999. 57 p.

WÄCHTER, K. Konstruktionslehre für Maschineningenieure. Berlin: Verlag Technik, 1987. 849 p. 